\title{
Traveling Wave Solutions for a Delayed SIRS Infectious Disease Model with Nonlocal Diffusion and Nonlinear Incidence
}

\author{
Xiaohong Tian and Rui Xu \\ Institute of Applied Mathematics, Shijiazhuang Mechanical Engineering College, No. 97 Heping West Road, \\ Shijiazhuang, Hebei 050003, China \\ Correspondence should be addressed to Xiaohong Tian; tianxh-2008@163.com
}

Received 17 February 2014; Accepted 8 March 2014; Published 10 April 2014

Academic Editor: Weiming Wang

Copyright (C) 2014 X. Tian and R. Xu. This is an open access article distributed under the Creative Commons Attribution License, which permits unrestricted use, distribution, and reproduction in any medium, provided the original work is properly cited.

A delayed SIRS infectious disease model with nonlocal diffusion and nonlinear incidence is investigated. By constructing a pair of upper-lower solutions and using Schauder's fixed point theorem, we derive the existence of a traveling wave solution connecting the disease-free steady state and the endemic steady state.

\section{Introduction}

Mathematical modeling has been proven to be valuable in studying the transmission dynamics of infectious diseases in a host population. We note that in disease progression, the spatial content of the environment plays a crucial role; the spread of germs, bacteria, and pathogen in the area is the main reason which leads to the spread of infectious disease. Thus, due to the large mobility of people within a country or even worldwide, spatially uniform models are not sufficient to give a realistic picture of a disease's diffusion. Considering the spatial effects, Gan et al. [1] considered the following SIRS epidemic model with spatial diffusion and time delay:

$$
\begin{gathered}
\frac{\partial S}{\partial t}=D_{S} \frac{\partial^{2} S}{\partial x^{2}}+A-d S(x, t) \\
-\beta S(x, t) I(x, t-\tau)+\delta R(x, t), \\
\frac{\partial I}{\partial t}=D_{I} \frac{\partial^{2} I}{\partial x^{2}}+\beta S(x, t) I(x, t-\tau)-(d+\gamma+a) I(x, t), \\
\frac{\partial R}{\partial t}=D_{R} \frac{\partial^{2} R}{\partial x^{2}}+\gamma I(x, t)-(d+\delta) R(x, t),
\end{gathered}
$$

where $S(t)$ represents the number of individuals who are susceptible to the disease, $I(t)$ represents the number of infected individuals who are infectious and are able to spread the disease by contact with susceptible individuals, and $R(t)$ represents the number of individuals who have been removed from the possibility of infection through full immunity. The parameters $A, a, d, \beta, \gamma, \delta$ are positive constants in which $A$ is the recruitment rate of the population, $a$ is the death rate due to disease, $d$ is the natural death rate of the population, $\beta$ is the transmission rate, $\gamma$ is the recovery rate of the infective individuals, and $\delta$ is the rate at which recovered individuals lose immunity and return to the susceptible class. $\tau>0$ is a fixed time during which the infectious agents develop in the vector and it is only after that time that the infected vector can infect a susceptible human. $D_{S}, D_{I}$, and $D_{R}$ denote the corresponding diffusion rates for the susceptible, infected, and removed populations, respectively. In [1], by constructing a pair of upper-lower solutions, the existence of a traveling wave solution connecting the disease-free steady state and the endemic steady state was given. In recent years, there has been a fair amount of work on epidemiological models with spatial diffusion (see, e.g., [2-6]).

In system (1), the Laplacian operator $\partial^{2} / \partial x^{2}$ has been used to model the diffusion of the species, which suggests that the population at the location $x$ can only be influenced by the variation of the population near the location $x$. However, in dynamics of infectious diseases, dispersal is better described as a long range process rather than as a local one. At the same time, studies of disease infections have also shown that 
reaction-diffusion equation does not accurately describe the spatial and temporal behavior of some diseases, for example, in the incubation period of SARS patients, who can move freely and the movement may transmit the disease to other people. Since the long range effect is taken into account, nonlocal diffusion equations have received great interest and have been recently intensively studied to analyze the long range effects of the dispersal (see, e.g., [7-12]). A basic nonlocal diffusion equation is of the form [13]

$$
\frac{\partial}{\partial t} u(x, t)=J * u-u(x, t)+f(u)
$$

where the kernel $J$ of the convolution $(J * u)(x, t)=\int_{\mathbb{R}} J(x-$ $y) u(y, t) d y$ is a nonnegative function of mass one and $f$ a given nonlinearity. As stated in [9], if $u(x, t)$ represents the density of a species at the point $x$ and time $t$ and $J(x-y)$ is regarded as the probability distribution of jumping from location $y$ to location $x$, then $\int_{\mathbb{R}} J(x-y) u(y, t) d y$ is the rate at which individuals arrive at position $x$ from all other places and $-u(x, t)=-\int_{\mathbb{R}} J(x-y) u(y, t) d y$ is the rate at which they leave location $x$ to travel to all other sites. The diffusion is modeled by a convolution operator which looks to be biologically reasonable.

We note that in system (1), Gan et al. used a bilinear incidence rate $\beta S I$ based on the law of mass action. If the number of susceptible individuals is very large, it is unreasonable to consider the bilinear incidence within a certain limited time, because the number of effective contacts between infective individuals and susceptible individuals may saturate at high infective levels due to crowding of infective individuals or due to the protection measures by the susceptible individuals. After a study of the cholera epidemic spread in Bari in 1973, Capasso and Serio [14] introduced a saturated incidence rate $g(I) S$ into epidemic models, where $g(I)$ tends to a saturation level when $I$ gets large; that is, $g(I)=\beta I /(1+\alpha I)$; here $\beta I$ measures the force of infection of the disease, and $1 /(1+\alpha I)$ measures the inhibition effect from the behavioral change of the susceptible individuals when their number increases or from the crowding effect of the susceptible individuals. This incidence rate seems more reasonable than the bilinear incidence rate, because it includes the behavioral change and crowding effect of the infective individuals and prevents the unboundedness of the contact rate by choosing suitable parameters [15].

Motivated by the works of Capasso and Serio [14], Gan et al. [1], and $\mathrm{Li}$ et al. [13], in this paper, we study the following delayed SIRS infectious disease model with nonlocal diffusion:

$$
\begin{aligned}
\frac{\partial S}{\partial t}= & D[(J * S)(x, t)-S(x, t)]+A-d S(x, t) \\
& -\frac{\beta S(x, t) I(x, t-\tau)}{1+\alpha I(x, t-\tau)}+\delta R(x, t),
\end{aligned}
$$

$$
\begin{aligned}
\frac{\partial I}{\partial t}= & D[(J * I)(x, t)-I(x, t)]+\frac{\beta S(x, t) I(x, t-\tau)}{1+\alpha I(x, t-\tau)} \\
& -(d+\gamma+a) I(x, t), \\
\frac{\partial R}{\partial t}= & D[(J * R)(x, t)-R(x, t)]+\gamma I(x, t) \\
& -(d+\delta) R(x, t),
\end{aligned}
$$

where the parameter $D$ denotes the corresponding diffusion rates for the three populations, respectively. Here, for simplicity, we assume $D_{S}=D_{I}=D_{R}=D . J(z)$ is a kernel function which is continuous satisfying

(A1) $\int_{\mathbb{R}} J(x) d x=1, J(x) \geq 0$ and $J(x)=J(-x)$, for $x \in \mathbb{R}$. For any fixed $\mu>0, J_{\mu}:=\int_{-\infty}^{+\infty} J(x) e^{\mu|x|} d x<\infty$ and

$$
\lim _{\mu \rightarrow \infty} \int_{-\infty}^{+\infty} J(x) e^{\mu|x|} d x=\infty .
$$

The initial conditions for system (3) take the form

$$
\begin{array}{r}
S(x, t)=\rho_{1}(x, t), \quad I(x, t)=\rho_{2}(x, t), \quad R(x, t)=\rho_{3}(x, t), \\
t \in[-\tau, 0] .
\end{array}
$$

In the biological context, it is important to analyse the epidemic waves which are described by traveling wave solutions propagating with a certain speed. In this paper, our focus is on the existence of traveling wave solutions to the SIRS infectious disease model (3).

The rest of this paper is organized as follows. In Section 2, by constructing a pair of upper-lower solutions and using Schauder's fixed point theorem, the existence of traveling wave solutions connecting the disease-free steady state and the endemic steady state of system (3) is established. In Section 3, a brief concluding remark is given to end this work.

\section{Existence of Traveling Waves}

In this section, we apply Schauder's fixed point theorem, the method of cross-iteration scheme associated with upperlower solutions, to study the existence of traveling wave solutions of system (3) connecting the disease-free steady state and the endemic steady state.

Denote

$$
\mathscr{R}_{0}=\frac{A \beta}{d(d+\gamma+a)} .
$$

$\mathscr{R}_{0}$ is called the basic reproduction ratio of system (3), which describes the average number of newly infected cells generated from one infected cell at the beginning of the infectious process. This quantity determines the thresholds for disease transmissions. It is easy to show that system (3) always has a disease-free steady state $E^{0}(A / d, 0,0)$. Further, 
if $\mathscr{R}_{0}>1$, system (3) has a unique endemic steady state $E^{*}\left(S^{*}, I^{*}, R^{*}\right)$, where

$$
\begin{aligned}
S^{*} & =\frac{(d+\gamma+a)\left(1+\alpha I^{*}\right)}{\beta}, \\
I^{*} & =\frac{d(d+\delta)(d+\gamma+a)\left(\mathscr{R}_{0}-1\right)}{(\alpha d+\beta)(d+\delta)(d+\gamma+a)-\beta \delta \gamma}, \\
R^{*} & =\frac{\gamma}{d+\delta} I^{*}
\end{aligned}
$$

Denoting $N=S+I+R$, then system (3) is equivalent to the following system:

$$
\begin{aligned}
\frac{\partial N}{\partial t}= & D[(J * N)(x, t)-N(x, t)]+A-d N(x, t) \\
& -a I(x, t), \\
\frac{\partial I}{\partial t}= & D[(J * I)(x, t)-I(x, t)] \\
& +\frac{\beta[N(x, t)-I(x, t)-R(x, t)] I(x, t-\tau)}{1+\alpha I(x, t-\tau)} \\
& -(d+\gamma+a) I(x, t), \\
\frac{\partial R}{\partial t}= & D[(J * R)(x, t)-R(x, t)]+\gamma I(x, t) \\
& -(d+\delta) R(x, t) .
\end{aligned}
$$

By making a change of variables $\widetilde{N}=A / d-N, \widetilde{I}=I, \widetilde{R}=$ $R$ and dropping the tildes, system (8) becomes

$$
\begin{aligned}
\frac{\partial N}{\partial t}= & D[(J * N)(x, t)-N(x, t)]-d N(x, t)+a I(x, t), \\
\frac{\partial I}{\partial t}= & D[(J * I)(x, t)-I(x, t)] \\
& +\frac{\beta[A / d-N(x, t)-I(x, t)-R(x, t)] I(x, t-\tau)}{1+\alpha I(x, t-\tau)} \\
& -(d+\gamma+a) I(x, t), \\
& \frac{\partial R}{\partial t}=D[(J * R)(x, t)-R(x, t)]+\gamma I(x, t) \\
& -(d+\delta) R(x, t) .
\end{aligned}
$$

It is easy to show that if $\mathscr{R}_{0}>1$, system (9) has two steady states $(0,0,0)$ and $\left(k_{1}, k_{2}, k_{3}\right)$, where $k_{1}=A / d-S^{*}-I^{*}-R^{*}$ and $k_{2}=I^{*}, k_{3}=R^{*}$.

A traveling wave solution of (9) is a special translation invariant solution of the form $(N(x, t), I(x, t), R(x, t))=$ $(\phi(x+c t), \varphi(x+c t), \psi(x+c t))$, where $(\phi, \varphi, \psi) \in C\left(\mathbb{R}, \mathbb{R}^{3}\right)$ is the profile of the wave that propagates through onedimensional spatial domain at a constant speed $c>0$. On substituting $N(x, t)=\phi(x+c t), I(x, t)=\varphi(x+c t)$,
$R(x, t)=\psi(x+c t)$ into (9) and denoting the traveling wave coordinate $x+c t$ still by $t$, we derive from (9) that

$$
\begin{aligned}
& c \phi^{\prime}(t)=D \int_{R} J(t-y) \phi(y) d y-D \phi(t)+f_{c 1}\left(\phi_{t}, \varphi_{t}, \psi_{t}\right), \\
& c \varphi^{\prime}(t)=D \int_{R} J(t-y) \varphi(y) d y-D \phi(t)+f_{c 2}\left(\phi_{t}, \varphi_{t}, \psi_{t}\right), \\
& c \psi^{\prime}(t)=D \int_{R} J(t-y) \psi(y) d y-D \psi(t)+f_{c 3}\left(\phi_{t}, \varphi_{t}, \psi_{t}\right),
\end{aligned}
$$

where

$$
\begin{gathered}
f_{c 1}\left(\phi_{t}, \varphi_{t}, \psi_{t}\right)=-d \phi(t)+a \varphi(t), \\
f_{c 2}\left(\phi_{t}, \varphi_{t}, \psi_{t}\right) \\
=\frac{\beta[A / d-\phi(t)-\varphi(t)-\psi(t)] \varphi(t-c \tau)}{1+\alpha \varphi(t-c \tau)} \\
-(d+\gamma+a) \varphi(t), \\
f_{c 3}\left(\phi_{t}, \varphi_{t}, \psi_{t}\right)=\gamma \varphi(t)-(d+\delta) \psi(t) .
\end{gathered}
$$

Equation (10) will be solved subject to the following boundary value conditions:

$$
\begin{aligned}
& \lim _{t \rightarrow-\infty}(\phi(t), \varphi(t), \psi(t))=(0,0,0), \\
& \lim _{t \rightarrow+\infty}(\phi(t), \varphi(t), \psi(t))=\left(k_{1}, k_{2}, k_{3}\right) .
\end{aligned}
$$

Now, we give the definition of upper and lower solutions of system (10) as follows.

Definition 1. A pair of continuous functions $\bar{\Phi}=(\bar{\phi}, \bar{\varphi}, \bar{\psi})$ and $\underline{\Phi}=(\phi, \varphi, \psi)$ are called a pair of upper-lower solutions of system (10), if there exist constants $T_{i}(i=1,2, \ldots, m)$ such that $\bar{\Phi}$ and $\underline{\Phi}$ are twice differential on $\mathbb{R} \backslash\left\{T_{i}: i=1,2, \ldots, m\right\}$ and satisfy

$$
\begin{gathered}
D \int_{R} J(t-y) \bar{\phi}(y) d y-D \bar{\phi}(t)-c \bar{\phi}^{\prime}(t) \\
\quad+f_{c 1}\left(\bar{\phi}_{t}, \bar{\varphi}_{t}, \bar{\psi}_{t}\right) \leq 0, \\
D \int_{R} J(t-y) \bar{\varphi}(y) d y-D \bar{\varphi}(t)-c \bar{\varphi}^{\prime}(t) \\
\quad+f_{c 2}\left(\underline{\phi}_{t}, \bar{\varphi}_{t}, \underline{\psi}_{t}\right) \leq 0, \\
D \int_{R} J(t-y) \bar{\psi}(y) d y-D \bar{\psi}(t)-c \bar{\psi}^{\prime}(t) \\
\quad+f_{c 3}\left(\bar{\phi}_{t}, \bar{\varphi}_{t}, \bar{\psi}_{t}\right) \leq 0,
\end{gathered}
$$




$$
\begin{gathered}
D \int_{R} J(t-y) \underline{\phi}(y) d y-D \underline{\phi}(t)-c \underline{\phi}^{\prime}(t) \\
+f_{c 1}\left(\underline{\phi}_{t}, \underline{\varphi}_{t}, \underline{\psi}_{t}\right) \geq 0 \\
D \int_{R} J(t-y) \underline{\varphi}(y) d y-D \underline{\varphi}(t)-c \underline{\varphi}^{\prime}(t) \\
+f_{c 2}\left(\bar{\phi}_{t}, \underline{\varphi}_{t}, \bar{\psi}_{t}\right) \geq 0 \\
D \int_{R} J(t-y) \underline{\psi}(y) d y-D \underline{\psi}(t)-c \underline{\psi}^{\prime}(t) \\
+f_{c 3}\left(\underline{\phi}_{t}, \underline{\varphi}_{t}, \underline{\psi}_{t}\right) \geq 0,
\end{gathered}
$$

for $t \in \mathbb{R} \backslash\left\{T_{i}: i=1,2, \ldots, m\right\}$.

In what follows, we assume that there exist an upper solution $\bar{\Phi}(t)=(\bar{\phi}, \bar{\varphi}, \bar{\psi})(t)$ and a lower solution $\underline{\Phi}(t)=$ $(\underline{\phi}, \underline{\varphi}, \underline{\psi})(t)$ of system (10) satisfying (P1)-(P2):

(P1) $0 \leq \underline{\Phi} \leq \bar{\Phi} \leq M=\left(M_{1}, M_{2}, M_{3}\right)$;

(P2) $\lim _{t \rightarrow-\infty} \bar{\Phi}(t)=\mathbf{0}, \lim _{t \rightarrow+\infty} \underline{\Phi}(t)=\lim _{t \rightarrow+\infty} \bar{\Phi}(t)=$ $\mathbf{K}=\left(k_{1}, k_{2}, k_{3}\right)$.

Let

$$
\begin{gathered}
C_{[0, M]}\left(\mathbb{R}, \mathbb{R}^{3}\right)=\left\{\Phi(t)=(\phi, \varphi, \psi)(t) \in C\left(\mathbb{R}, \mathbb{R}^{3}\right)\right. \\
: \mathbf{0} \leq \Phi(t) \leq M, t \in \mathbb{R}\},
\end{gathered}
$$

where $M_{i}>k_{i}(i=1,2,3)$ satisfy

$$
\begin{gathered}
\frac{A \beta}{d}-(\gamma+a)>a \frac{M_{2}}{M_{1}}>d, \\
\frac{A \beta}{d}-(\gamma+a)>\gamma \frac{M_{2}}{M_{3}}-\delta>d, \\
\frac{A}{d} \geq M_{1}+M_{2}+M_{3} .
\end{gathered}
$$

We look for traveling wave solutions to system (10) in the following profile set:

$$
\begin{aligned}
\Gamma=\left\{(\phi, \varphi, \psi)(t) \in C_{[0, M]}\left(\mathbb{R}, \mathbb{R}^{3}\right)\right. \\
\quad:(\underline{\phi}, \underline{\varphi}, \underline{\psi})(t) \leq(\phi, \varphi, \psi)(t) \leq(\bar{\phi}, \bar{\varphi}, \bar{\psi})(t)\} .
\end{aligned}
$$

Obviously, $\Gamma$ is nonempty, convex, closed, and bounded.

Furthermore, corresponding to (10), we make the following hypotheses.

(A2) There exist three positive constants $L_{i}>0(i=1,2,3)$ such that

$$
\left|f_{c i}\left(\phi_{1}, \varphi_{1}, \psi_{1}\right)-f_{c i}\left(\phi_{2}, \varphi_{2}, \psi_{2}\right)\right| \leq L_{i}\|\Phi(t)-\Psi(t)\|
$$

for $\Phi(t)=\left(\phi_{1}, \varphi_{1}, \psi_{1}\right)(t)$ and $\Psi(t)=\left(\phi_{2}, \varphi_{2}, \psi_{2}\right)(t) \epsilon$ $C\left([-\tau, 0], \mathbb{R}^{3}\right)$ with $(0,0,0) \leq\left(\phi_{j}(t), \varphi_{j}(t), \psi_{j}(t)\right) \leq$ $\left(M_{1}, M_{2}, M_{3}\right), j=1,2, t \in[-\tau, 0], M_{i} \geq k_{i}$, are positive constants.
For $\Phi=(\phi, \varphi, \psi) \in C_{[0, M]}\left(\mathbb{R}, \mathbb{R}^{3}\right)$, we define two operators $H=\left(H_{1}, H_{2}, H_{3}\right)$ and $F=\left(F_{1}, F_{2}, F_{3}\right)$ from $C_{[0, M]}\left(\mathbb{R}, \mathbb{R}^{3}\right)$ to $C\left(\mathbb{R}, \mathbb{R}^{3}\right)$ by

$$
\begin{aligned}
& H_{1}(\phi, \varphi, \psi)(t)=D \int_{R} J(t-y) \phi(y) d y+a \varphi(t), \\
& H_{2}(\phi, \varphi, \psi)(t) \\
& =D \int_{R} J(t-y) \varphi(y) d y+\beta M_{2} \varphi(t) \\
& \quad+\frac{\beta[A / d-\phi(t)-\varphi(t)-\psi(t)] \varphi(t-c \tau)}{1+\alpha \varphi(t-c \tau)},
\end{aligned}
$$

$$
H_{3}(\phi, \varphi, \psi)(t)=D \int_{R} J(t-y) \psi(y) d y+\gamma \varphi(t)
$$$$
F_{i}(\phi, \varphi, \psi)(t)=\frac{1}{c} e^{-\left(\beta_{i} / c\right) t} \int_{-\infty}^{t} e^{\left(\beta_{i} / c\right) s} H_{i}(\phi, \varphi, \psi)(s) d s
$$

Letting $\beta_{1}=D+d, \beta_{2}=D+d+\gamma+a$, and $\beta_{3}=D+d+\delta$, then system (10) can be rewritten as

$$
\begin{aligned}
& c \phi^{\prime}(t)=-\beta_{1} \phi(t)+H_{1}(\phi, \varphi, \psi)(t), \\
& c \varphi^{\prime}(t)=-\beta_{2} \varphi(t)+H_{2}(\phi, \varphi, \psi)(t), \\
& c \psi^{\prime}(t)=-\beta_{3} \psi(t)+H_{3}(\phi, \varphi, \psi)(t),
\end{aligned}
$$

and then $F$ is well defined such that

$$
\begin{array}{r}
c F_{i}^{\prime}(\phi, \varphi, \psi)(t)=-\beta_{i} F_{i}(\phi, \varphi, \psi)(t)+H_{i}(\phi, \varphi, \psi)(t) \\
(i=1,2,3) .
\end{array}
$$

Hence, a fixed point of $F$ is a solution of (10), which is a traveling wave solution of $(9)$ connecting $\mathbf{0}=(0,0,0)$ with $\mathbf{K}=\left(k_{1}, k_{2}, k_{3}\right)$ if it satisfies (P2).

In the following, we introduce some lemmas to support our main results.

For $\mu>0$, define

$$
\begin{gathered}
B_{\mu}\left(\mathbb{R}, \mathbb{R}^{3}\right)=\left\{(\phi, \varphi, \psi) \in C\left(\mathbb{R}, \mathbb{R}^{3}\right)\right. \\
\left.\quad: \sup _{t \in \mathbb{R}}|(\phi, \varphi, \psi)(t)| e^{-\mu|t|}<\infty\right\}, \\
|(\phi, \varphi, \psi)(t)|_{\mu}=\sup _{t \in \mathbb{R}}|(\phi, \varphi, \psi)(t)| e^{-\mu|t|} .
\end{gathered}
$$

Then it is easy to check that $\left(B_{\mu}\left(\mathbb{R}, \mathbb{R}^{3}\right),|\cdot|\right)$ is a Banach space. In view of the definition of $H$ and $F$, we can easily see that they admit the following properties. 
Lemma 2. Let $\left(A_{1}\right)$ hold. One has

(i)

$$
\begin{gathered}
H_{1}(\phi, \varphi, \psi)(t) \geq 0, \quad H_{3}(\phi, \varphi, \psi)(t) \geq 0, \\
H_{1}\left(\phi_{2}, \varphi_{2}, \psi_{2}\right)(t) \leq H_{1}\left(\phi_{1}, \varphi_{1}, \psi_{1}\right)(t), \\
H_{2}\left(\phi_{1}, \varphi_{1}, \psi_{1}\right)(t) \leq H_{2}\left(\phi_{2}, \varphi_{1}, \psi_{1}\right)(t), \\
H_{2}\left(\phi_{1}, \varphi_{2}, \psi_{1}\right)(t) \leq H_{2}\left(\phi_{1}, \varphi_{1}, \psi_{1}\right)(t), \\
H_{2}\left(\phi_{1}, \varphi_{1}, \psi_{1}\right)(t) \leq H_{2}\left(\phi_{1}, \varphi_{1}, \psi_{2}\right)(t), \\
H_{3}\left(\phi_{2}, \varphi_{2}, \psi_{2}\right)(t) \leq H_{3}\left(\phi_{1}, \varphi_{1}, \psi_{1}\right)(t),
\end{gathered}
$$

(ii)

$$
\begin{aligned}
& F_{1}\left(\phi_{2}, \varphi_{2}, \psi_{2}\right)(t) \leq F_{1}\left(\phi_{1}, \varphi_{1}, \psi_{1}\right)(t), \\
& F_{2}\left(\phi_{1}, \varphi_{1}, \psi_{1}\right)(t) \leq F_{2}\left(\phi_{2}, \varphi_{1}, \psi_{1}\right)(t), \\
& F_{2}\left(\phi_{1}, \varphi_{2}, \psi_{1}\right)(t) \leq F_{2}\left(\phi_{1}, \varphi_{1}, \psi_{1}\right)(t), \\
& F_{2}\left(\phi_{1}, \varphi_{1}, \psi_{1}\right)(t) \leq F_{2}\left(\phi_{1}, \varphi_{1}, \psi_{2}\right)(t), \\
& F_{3}\left(\phi_{2}, \varphi_{2}, \psi_{2}\right)(t) \leq F_{3}\left(\phi_{1}, \varphi_{1}, \psi_{1}\right)(t),
\end{aligned}
$$

for $t \in \mathbb{R}$ with $0 \leq \phi_{2}(t) \leq \phi_{1}(t) \leq M_{1}, 0 \leq \varphi_{2}(t) \leq \varphi_{1}(t) \leq$ $M_{2}, 0 \leq \psi_{2}(t) \leq \psi_{1}(t) \leq M_{3}$.

By using a similar argument as in the proof of Lemmas 3.3-3.6 in [16], one can show the following lemmas.

Lemma 3. Assume that (A2) holds. $F=\left(F_{1}, F_{2}, F_{3}\right)$ is continuous with respect to the norm $|\cdot|_{\mu}$ in $B_{\mu}\left(\mathbb{R}, \mathbb{R}^{3}\right)$.

Lemma 4. $F(\Gamma((\underline{\phi}, \underline{\varphi}, \underline{\psi}),(\bar{\phi}, \bar{\varphi}, \bar{\psi}))) \subset \Gamma((\underline{\phi}, \underline{\varphi}, \underline{\psi}),(\bar{\phi}, \bar{\varphi}, \bar{\psi}))$, where $F=\left(F_{1}, F_{2}, F_{3}\right)$.

Lemma 5. $F: \Gamma((\underline{\phi}, \underline{\varphi}, \underline{\psi}),(\bar{\phi}, \bar{\varphi}, \bar{\psi})) \rightarrow \Gamma((\underline{\phi}, \underline{\varphi}, \underline{\psi}),(\bar{\phi}, \bar{\varphi}, \bar{\psi}))$ is compact.

We now consider the following equations:

$$
\begin{aligned}
& \Delta_{1}(\lambda, c):=D \int_{\mathbb{R}} J(y) e^{-\lambda y} d y-D-c \lambda-d+a \frac{M_{2}}{M_{1}} \\
& \Delta_{2}(\lambda, c):=D \int_{\mathbb{R}} J(y) e^{-\lambda y} d y-D-c \lambda+\frac{A \beta}{d}-(d+\gamma+a), \\
& \Delta_{3}(\lambda, c):=D \int_{\mathbb{R}} J(y) e^{-\lambda y} d y-D-c \lambda-(d+\delta)+\gamma \frac{M_{2}}{M_{3}} .
\end{aligned}
$$

Since $(A 1)$ and (15) hold, direct calculations show that

$$
\begin{array}{cc}
\Delta_{1}(0, c)=-d+a \frac{M_{2}}{M_{1}}>0, & \Delta_{1}(\lambda,+\infty)=-\infty, \\
\forall \lambda>0 ; & \forall \lambda>0 ; \\
\frac{\partial \Delta_{1}(\lambda, c)}{\partial c}=-\lambda<0, \quad \forall \lambda>0 & \\
\frac{\partial^{2} \Delta_{1}(\lambda, c)}{\partial \lambda^{2}}=D \int_{\mathbb{R}} y^{2} J(y) e^{-\lambda y} d y>0 .
\end{array}
$$

Therefore, we obtain that there exist $c_{1}^{*}>0$ and $\lambda_{1 *}>0$ such that $\partial \Delta_{1}(\lambda, c) /\left.\partial \lambda\right|_{\left(\lambda_{1 *}, c_{1}^{*}\right)}=0$ and $\Delta_{1}\left(\lambda_{1 *}, c_{1}^{*}\right)=0$. Further, if $c>c_{1}^{*}$, there exist $\lambda_{1}(c)>0$ and $\lambda_{2}(c)>0$ satisfying

$$
0<\lambda_{1}(c)<\lambda_{1 *}<\lambda_{2}(c) \text {. }
$$

Similarly, we can show that there exist $c_{i}^{*}, \lambda_{i *}>0$ such that $\Delta_{i}\left(\lambda_{i *}, c_{i}^{*}\right)=0$. If $c>c_{i}^{*}$, there exist $\lambda_{j}(c)>0$ satisfying $0<\lambda_{3}(c)<\lambda_{2 *}<\lambda_{4}(c)$ and $0<\lambda_{5}(c)<\lambda_{3 *}<\lambda_{6}(c)(i=$ $2,3 ; j=3,4,5,6)$.

Lemma 6. Let $c^{*}=\max \left\{c_{1}^{*}, c_{2}^{*}, c_{3}^{*}\right\}$. Assume that $\mathscr{R}_{0}>1$; then one has $\lambda_{1}(c)<\lambda_{3}(c)$ and $\lambda_{5}(c)<\lambda_{3}(c)$.

Proof. Define

$$
\begin{aligned}
& h(\lambda)=D \int_{\mathbb{R}} J(y) e^{-\lambda y} d y, \\
& g_{1}(\lambda)=c \lambda+D+d-a \frac{M_{2}}{M_{1}}, \\
& g_{2}(\lambda)=c \lambda+D+d+\gamma+a-\frac{A \beta}{d}, \\
& g_{3}(\lambda)=c \lambda+D+d+\delta-\gamma \frac{M_{2}}{M_{3}} .
\end{aligned}
$$

It is easy to show that

$$
\begin{gathered}
h\left(\lambda_{1}\right)=g_{1}\left(\lambda_{1}\right), \quad h\left(\lambda_{3}\right)=g_{2}\left(\lambda_{3}\right), \\
h\left(\lambda_{5}\right)=g_{3}\left(\lambda_{5}\right) .
\end{gathered}
$$

If $\mathscr{R}_{0}>1$, then by (9), we see that $g_{2}(0)<g_{1}(0)$ and $g_{2}(0)<$ $g_{3}(0)$. Note that $h^{\prime \prime}(\lambda)>0$ for all $\lambda$; hence, we have $\lambda_{1}(c)<$ $\lambda_{3}(c)$ and $\lambda_{5}(c)<\lambda_{3}(c)$. 
Suppose that $\mathscr{R}_{0}>1$ and $a / d+\gamma /(d+\delta)<1$; we can choose $\varepsilon_{i}>0(i=1,2, \ldots, 6)$, and $\varepsilon_{1}, \varepsilon_{2} \in\left(0, k_{1}\right), \varepsilon_{3}, \varepsilon_{4} \in$ $\left(0, k_{2}\right), \varepsilon_{5}, \varepsilon_{6} \in\left(0, k_{3}\right)$ satisfying

$$
\begin{gathered}
a\left(k_{2}+\varepsilon_{3}\right)-d\left(k_{1}+\varepsilon_{1}\right)<0, \\
\beta\left(\frac{A}{d}-k_{1}+\varepsilon_{2}-k_{2}-\varepsilon_{3}-k_{3}+\varepsilon_{6}\right) \\
<(d+\gamma+a)\left(1+\alpha\left(k_{2}+\varepsilon_{3}\right)\right), \\
\gamma\left(k_{2}+\varepsilon_{3}\right)-(d+\delta)\left(k_{3}+\varepsilon_{5}\right)<0, \\
-d\left(k_{1}-\varepsilon_{2}\right)+a\left(k_{2}-\varepsilon_{4}\right)>0, \\
\beta\left(\frac{A}{d}-k_{1}-\varepsilon_{1}-k_{2}+\varepsilon_{4}-k_{3}-\varepsilon_{5}\right) \\
>(d+\gamma+a)\left(1+\alpha\left(k_{2}-\varepsilon_{4}\right)\right), \\
\gamma\left(k_{2}-\varepsilon_{4}\right)-(d+\delta)\left(k_{3}-\varepsilon_{6}\right)>0 .
\end{gathered}
$$

In fact, noting that $d k_{1}=a k_{2}$, for $\varepsilon_{3}, \varepsilon_{4} \in\left(0, k_{2}\right)$, there exist $\varepsilon_{1}, \varepsilon_{2} \in\left(0, k_{1}\right)$ and $\varepsilon_{5}, \varepsilon_{6} \in\left(0, k_{3}\right)$ such that

$$
\begin{aligned}
& k_{1}>\varepsilon_{1}>\frac{a}{d} \varepsilon_{3}=\frac{a}{d} \varepsilon_{3}+\frac{a}{d} k_{2}-k_{1}, \\
& k_{1}>\varepsilon_{2}>\frac{a}{d} \varepsilon_{4}=\frac{a}{d} \varepsilon_{4}+k_{1}-\frac{a}{d} k_{2},
\end{aligned}
$$

which yield

$$
\begin{gathered}
a\left(k_{2}+\varepsilon_{3}\right)-d\left(k_{1}+\varepsilon_{1}\right)<0, \\
-d\left(k_{1}-\varepsilon_{2}\right)+a\left(k_{2}-\varepsilon_{4}\right)>0 .
\end{gathered}
$$

Since $\gamma k_{2}=(d+\delta) k_{3}$, for $\varepsilon_{3}, \varepsilon_{4} \in\left(0, k_{2}\right)$, we can find $\varepsilon_{1}, \varepsilon_{2} \in\left(0, k_{1}\right)$ and $\varepsilon_{5}, \varepsilon_{6} \in\left(0, k_{3}\right)$ such that

$$
\begin{aligned}
k_{3} & >\varepsilon_{5}>\frac{\gamma}{d+\delta} \varepsilon_{3}=\frac{\gamma}{d+\delta} \varepsilon_{3}+\frac{\gamma}{d+\delta} k_{2}-k_{3} \\
& \Longrightarrow \gamma\left(k_{2}+\varepsilon_{3}\right)-(d+\delta)\left(k_{3}+\varepsilon_{5}\right)<0, \\
k_{3} & >\varepsilon_{6}>\frac{\gamma}{d+\delta} \varepsilon_{4}=\frac{\gamma}{d+\delta} \varepsilon_{4}+k_{3}-\frac{\gamma}{d+\delta} k_{2} \\
& \Longrightarrow \gamma\left(k_{2}-\varepsilon_{4}\right)-(d+\delta)\left(k_{3}-\varepsilon_{6}\right)>0 .
\end{aligned}
$$

If $a / d+\gamma /(d+\delta)<1$, then we can choose suitable values of $\varepsilon_{3}, \varepsilon_{4} \in\left(0, k_{2}\right)$ such that

$$
\left(\frac{a}{d}+\frac{\gamma}{d+\delta}\right) \varepsilon_{3}<\varepsilon_{4}, \quad\left(\frac{a}{d}+\frac{\gamma}{d+\delta}\right) \varepsilon_{4}<\varepsilon_{3} .
$$

Furthermore, we can choose $\varepsilon_{1}, \varepsilon_{2} \in\left(0, k_{1}\right), \varepsilon_{3}, \varepsilon_{4} \in$ $\left(0, k_{2}\right), \varepsilon_{5}, \varepsilon_{6} \in\left(0, k_{3}\right)$ satisfying

$$
\begin{aligned}
& \varepsilon_{2}>\frac{a}{d} \varepsilon_{4}, \quad \varepsilon_{6}>\frac{\gamma}{d+\delta} \varepsilon_{4}, \quad \frac{a}{d} \varepsilon_{4}+\frac{\gamma}{d+\delta} \varepsilon_{4}<\varepsilon_{3}, \\
& \varepsilon_{1}>\frac{a}{d} \varepsilon_{3}, \quad \varepsilon_{5}>\frac{\gamma}{d+\delta} \varepsilon_{3}, \quad \frac{a}{d} \varepsilon_{3}+\frac{\gamma}{d+\delta} \varepsilon_{3}<\varepsilon_{4} .
\end{aligned}
$$

Accordingly, there exist suitable constants $\varepsilon_{i}>0(i=$ $1, \ldots, 6)$ such that

$$
\varepsilon_{2}+\varepsilon_{6}-\varepsilon_{3}<0, \quad \varepsilon_{1}+\varepsilon_{5}-\varepsilon_{4}<0 .
$$

By the second equation of system (10), we have $\beta\left(A / d-k_{1}-\right.$ $\left.k_{2}-k_{3}\right)-(d+\gamma+a)\left(1+\alpha k_{2}\right)=0$. It then follows from (35) that

$$
\begin{gathered}
\beta\left(\frac{A}{d}-k_{1}+\varepsilon_{2}-k_{2}-\varepsilon_{3}-k_{3}+\varepsilon_{6}\right) \\
<(d+\gamma+a)\left(1+\alpha\left(k_{2}+\varepsilon_{3}\right)\right), \\
\beta\left(\frac{A}{d}-k_{1}-\varepsilon_{1}-k_{2}+\varepsilon_{4}-k_{3}-\varepsilon_{5}\right) \\
>(d+\gamma+a)\left(1+\alpha\left(k_{2}-\varepsilon_{4}\right)\right) .
\end{gathered}
$$

Now, we define the continuous functions $\bar{\Phi}(t)=$ $(\bar{\phi}(t), \bar{\varphi}(t), \bar{\psi}(t))$ and $\underline{\Phi}(t)=(\phi(t), \underline{\varphi}(t), \underline{\psi}(t))$ as follows:

$$
\begin{aligned}
& \bar{\phi}(t)= \begin{cases}k_{1} e^{\lambda_{1} t}, & t \leq t_{1}, \\
k_{1}+\varepsilon_{1} e^{-\lambda t}, & t>t_{1},\end{cases} \\
& \underline{\phi}(t)= \begin{cases}0, & t \leq t_{2}, \\
k_{1}-\varepsilon_{2} e^{-\lambda t}, & t>t_{2},\end{cases} \\
& \bar{\varphi}(t)= \begin{cases}l k_{2} e^{\lambda_{3} t}, & t \leq t_{3}, \\
k_{2}+\varepsilon_{3} e^{-\lambda t}, & t>t_{3},\end{cases} \\
& \underline{\varphi}(t)= \begin{cases}0, & t \leq t_{4}, \\
k_{2}-\varepsilon_{4} e^{-\lambda t}, & t>t_{4},\end{cases} \\
& \bar{\psi}(t)= \begin{cases}k_{3} e^{\lambda_{5} t}, & t \leq t_{5}, \\
k_{3}+\varepsilon_{5} e^{-\lambda t}, & t>t_{5},\end{cases} \\
& \underline{\psi}(t)= \begin{cases}0, & t \leq t_{6}, \\
k_{3}-\varepsilon_{6} e^{-\lambda t}, & t>t_{6},\end{cases}
\end{aligned}
$$

where $t_{1}, t_{3}, t_{5}>0, t_{2}, t_{4}, t_{6}<0$ and $\lambda>0$ is a constant sufficiently small to be chosen later. Then we can choose $\lambda>$ 0 to be sufficiently small such that $t_{1}>0, t_{3}>0, t_{5}>0$ satisfying

$$
\begin{aligned}
& k_{1}+\varepsilon_{1}>M_{1}=\sup _{t \in \mathbb{R}} \bar{\phi}(t)=k_{1} e^{\lambda_{1} t_{1}}>k_{1}, \\
& k_{2}+\varepsilon_{3}>M_{2}=\sup _{t \in \mathbb{R}} \bar{\varphi}(t)=k_{2} e^{\lambda_{3} t_{3}}>k_{2}, \\
& k_{3}+\varepsilon_{5}>M_{3}=\sup _{t \in \mathbb{R}} \bar{\psi}(t)=k_{3} e^{\lambda_{5} t_{5}}>k_{3},
\end{aligned}
$$

where $M_{1}, M_{2}, M_{3}$ are defined in (15). Furthermore, we can choose $l \in(0,1)$ such that $t_{3} \geq \max \left\{t_{1}, t_{5}\right\}$. If $a / d+\gamma /(\delta+d)<$ 1 , it is easy to show that $t_{4} \leq \min \left\{t_{2}, t_{6}\right\}$. Clearly, $\bar{\Phi}(t)$ and $\underline{\Phi}(t)$ satisfy (P1) and (P2).

Lemma 7. $\bar{\Phi}(t)=(\bar{\phi}(t), \bar{\varphi}(t), \bar{\psi}(t))$ is an upper solution of system (10). 
Proof. Denote

$$
\begin{aligned}
P_{1}(t)= & D \int_{\mathbb{R}} J(t-y) \bar{\phi}(y) d y-D \bar{\phi}(t)-c \bar{\phi}^{\prime}(t) \\
& -d \bar{\phi}(t)+a \bar{\varphi}(t), \\
P_{2}(t)= & D \int_{\mathbb{R}} J(t-y) \bar{\varphi}(y) d y-D \bar{\varphi}(t)-c \bar{\varphi}^{\prime}(t) \\
& +\frac{\beta[A / d-\underline{\phi}(t)-\bar{\varphi}(t)-\underline{\psi}(t)] \bar{\varphi}(t-c \tau)}{1+\alpha \bar{\varphi}(t-c \tau)} \\
& -(d+\gamma+a) \bar{\varphi}(t), \\
P_{3}(t)= & D \int_{\mathbb{R}} J(t-y) \bar{\psi}(y) d y-D \bar{\psi}(t)-c \bar{\psi}^{\prime}(t) \\
& +\gamma \bar{\varphi}(t)-(d+\delta) \bar{\psi}(t) .
\end{aligned}
$$

If $t \leq t_{1}, \bar{\phi}(t)=k_{1} e^{\lambda_{1} t}$ and $\bar{\varphi}(t)=l k_{2} e^{\lambda_{3} t}$. By Lemma 6, it follows that

$$
\begin{aligned}
& P_{1}(t)=k_{1} e^{\lambda_{1} t}\left[D \int_{\mathbb{R}} J(y) e^{-\lambda_{1} y} d y-D-c \lambda_{1}-d\right. \\
& \left.\quad+a l \frac{k_{2}}{k_{1}} e^{\left(\lambda_{3}-\lambda_{1}\right) t}\right] \\
& \leq k_{1} e^{\lambda_{1} t}\left[D \int_{\mathbb{R}} J(y) e^{-\lambda_{1} y} d y-D-c \lambda_{1}-d\right. \\
& \left.\quad+a \frac{M_{2}}{M_{1}}\right] \\
& \leq k_{1} e^{\lambda_{1} t} \Delta_{1}\left(\lambda_{1}, c\right)=0 .
\end{aligned}
$$

If $t_{1}<t \leq t_{3}, \bar{\phi}(t)=k_{1}+\varepsilon_{1} e^{-\lambda t}$ and $\bar{\varphi}(t)=l k_{2} e^{\lambda_{3} t}$. Then, we have

$$
\begin{aligned}
P_{1}(t)= & {\left[D \varepsilon_{1} \int_{\mathbb{R}} J(y) e^{\lambda y} d y-D \varepsilon_{1}+c \lambda \varepsilon_{1}\right] e^{-\lambda t} } \\
& -d\left(k_{1}+\varepsilon_{1} e^{-\lambda t}\right)+a l k_{2} e^{\lambda_{3} t} .
\end{aligned}
$$

Note that $l \in(0,1)$ and $a k_{2} / d=k_{1}$. Hence, for $\lambda$ sufficiently small, there exists $\lambda_{1}^{*}>0$ such that $P_{1}(t)<0$ for all $\lambda \epsilon$ $\left(0, \lambda_{1}^{*}\right)$.

If $t>t_{3}, \bar{\phi}(t)=k_{1}+\varepsilon_{1} e^{-\lambda t}$ and $\bar{\varphi}(t)=k_{2}+\varepsilon_{3} e^{-\lambda t}$. We obtain that

$$
\begin{aligned}
P_{1}(t) \leq & {\left[D \varepsilon_{1} \int_{\mathbb{R}} J(y) e^{\lambda y} d y-D \varepsilon_{1}+c \lambda \varepsilon_{1}\right] e^{-\lambda t} } \\
& -d\left(k_{1}+\varepsilon_{1} e^{-\lambda t}\right)+a\left(k_{2}+\varepsilon_{3} e^{-\lambda t}\right):=I_{1}(\lambda) .
\end{aligned}
$$

For $\lambda$ sufficiently small, $a\left(k_{2}+\varepsilon_{3}\right)<d\left(k_{1}+\varepsilon_{1}\right)$ implies that $I_{1}(0)<0$, and there exists $\lambda_{2}^{*}>0$ such that $P_{1}(t) \leq I_{1}(\lambda)<0$ for all $\lambda \in\left(0, \lambda_{2}^{*}\right)$.
If $t \leq t_{3}, \bar{\varphi}(t)=l k_{2} e^{\lambda_{3} t}$ and $\bar{\varphi}(t-c \tau)=l k_{2} e^{\lambda_{3}(t-c \tau)}$. It follows that

$$
\begin{gathered}
P_{2}(t) \leq l k_{2} e^{\lambda_{3} t}\left[D \int_{\mathbb{R}} J(y) e^{-\lambda_{3} y} d y-D-c \lambda_{3}+\frac{A \beta}{d}\right. \\
-(d+\gamma+a)] \\
=l k_{2} e^{\lambda_{3} t} \Delta_{2}\left(\lambda_{3}, c\right)=0 .
\end{gathered}
$$

If $t_{3}<t \leq t_{3}+c \tau, \bar{\varphi}(t)=k_{2}+\varepsilon_{3} e^{-\lambda t}, \bar{\varphi}(t-c \tau)=l k_{2} e^{\lambda_{3}(t-c \tau)}$, $\underline{\phi}(t)=k_{1}-\varepsilon_{2} e^{-\lambda t}$, and $\underline{\psi}(t)=k_{3}-\varepsilon_{6} e^{-\lambda t}$. We obtain that

$$
P_{2}(t)=\varepsilon_{3} e^{-\lambda t}\left[D \int_{\mathbb{R}} J(y) e^{\lambda y} d y-D+c \lambda\right]+I_{2}(\lambda),
$$

where

$$
\begin{aligned}
I_{2}(\lambda)= & \left(\beta \left[\frac{A}{d}-\left(k_{1}-\varepsilon_{2} e^{-\lambda t}\right)-\left(k_{2}+\varepsilon_{3} e^{-\lambda t}\right)\right.\right. \\
& \left.\left.-\left(k_{3}-\varepsilon_{6} e^{-\lambda t}\right)\right] l k_{2} e^{\lambda_{3}(t-c \tau)}\right) \\
& \times\left(1+\alpha l k_{2} e^{\lambda_{3}(t-c \tau)}\right)^{-1} \\
& -(d+\gamma+a)\left(k_{2}+\varepsilon_{3} e^{-\lambda \tau}\right) .
\end{aligned}
$$

Then by (29), we have

$$
\begin{aligned}
I_{2}(0)= & \frac{\beta\left(A / d-k_{1}+\varepsilon_{2}-k_{2}-\varepsilon_{3}-k_{3}+\varepsilon_{6}\right) l k_{2}}{1+\alpha l k_{2}} \\
& -(d+\gamma+a)\left(k_{2}+\varepsilon_{3}\right) \\
\leq & \frac{d+\gamma+a}{1+\alpha l k_{2}}\left((l-1) k_{2}-\varepsilon_{3}\right) .
\end{aligned}
$$

Since $l \in(0,1)$, for $\lambda$ sufficiently small, it is easy to show that $I_{2}(0)<0$ and there exists $\lambda_{3}^{*}>0$ such that $P_{2}(t)<0$ for all $\lambda \in\left(0, \lambda_{3}^{*}\right)$.

If $t>t_{3}+c \tau, \bar{\varphi}(t)=k_{2}+\varepsilon_{3} e^{-\lambda t}, \bar{\varphi}(t-c \tau)=k_{2}+\varepsilon_{3} e^{-\lambda(t-c \tau)}$, $\underline{\phi}(t)=k_{1}-\varepsilon_{2} e^{-\lambda t}$, and $\underline{\psi}(t)=k_{3}-\varepsilon_{6} e^{-\lambda t}$. It follows that

$$
P_{2}(t)=\varepsilon_{3} e^{-\lambda t}\left[D \int_{\mathbb{R}} J(y) e^{\lambda y} d y-D+c \lambda\right]+I_{3}(\lambda),
$$

where

$$
\begin{aligned}
I_{3}(\lambda)= & \left(\beta \left[\frac{A}{d}-\left(k_{1}-\varepsilon_{2} e^{-\lambda t}\right)-\left(k_{2}+\varepsilon_{3} e^{-\lambda t}\right)\right.\right. \\
& \left.\left.-\left(k_{3}-\varepsilon_{6} e^{-\lambda t}\right)\right]\left(k_{2}+\varepsilon_{3} e^{-\lambda(t-c \tau)}\right)\right) \\
& \times\left(1+\alpha\left(k_{2}+\varepsilon_{3} e^{-\lambda(t-c \tau)}\right)\right)^{-1} \\
& -(d+\gamma+a)\left(k_{2}+\varepsilon_{3} e^{-\lambda t}\right) .
\end{aligned}
$$

For $\lambda$ sufficiently small, by (29), we see that $I_{3}(0)<0$ and there exists $\lambda_{4}^{*}>0$ such that $P_{2}(t) \leq I_{3}(\lambda)<0$ for all $\lambda \epsilon$ $\left(0, \lambda_{4}^{*}\right)$. 
If $t \leq t_{5}, \bar{\psi}(t)=k_{3} e^{\lambda_{5} t}$ and $\bar{\varphi}(t)=l k_{2} e^{\lambda_{3}(t)}$. Then, by Lemma 6, we have

$$
\begin{gathered}
P_{3}(t)=k_{3} e^{\lambda_{5} t}\left[D \int_{\mathbb{R}} J(y) e^{-\lambda_{5} t} d y-D-c \lambda_{5}\right. \\
\left.-(d+\delta)+\frac{\gamma l k_{2} e^{\lambda_{3} t}}{k_{3} e^{\lambda_{5} t}}\right] \\
\leq k_{3} e^{\lambda_{5} t}\left[D \int_{\mathbb{R}} J(y) e^{-\lambda_{5} t} d y-D-c \lambda_{5}\right. \\
\left.-(d+\delta)+\frac{\gamma M_{2}}{M_{3}}\right] \\
=k_{3} e^{\lambda_{5} t} \Delta_{3}\left(\lambda_{5}, c\right)=0 .
\end{gathered}
$$

If $t_{5}<t \leq t_{3}, \bar{\psi}(t)=k_{3}+\varepsilon_{5} e^{-\lambda t}$ and $\bar{\varphi}(t)=l k_{2} e^{\lambda_{3} t}$. We derive that

$$
\begin{aligned}
P_{3}(t)= & \varepsilon_{3} e^{-\lambda t}\left[D \int_{\mathbb{R}} J(y) e^{\lambda y} d y-D+c \lambda\right]+\gamma l k_{2} e^{\lambda_{3} t} \\
& -(d+\delta)\left(k_{3}+\varepsilon_{5} e^{-\lambda t}\right):=I_{4}(\lambda) .
\end{aligned}
$$

Note that $l \in(0,1)$; then we have $l k_{2} e^{\lambda_{3} t} \leq k_{2} e^{\lambda_{3} t_{3}}<k_{2}+\varepsilon_{3}$. By (29), for $\lambda$ sufficiently small, it is easy to show that $I_{4}(0)<0$ and there exists $\lambda_{5}^{*}>0$ such that $P_{3}(t)<0$ for all $\lambda \in\left(0, \lambda_{5}^{*}\right)$.

If $t>t_{3}, \bar{\psi}(t)=k_{3}+\varepsilon_{5} e^{-\lambda t}$ and $\bar{\varphi}(t)=k_{2}+\varepsilon_{3} e^{-\lambda t}$. We obtain that

$$
\begin{aligned}
P_{3}(t) \leq & \varepsilon_{5} e^{-\lambda t}\left[D \int_{\mathbb{R}} J(y) e^{\lambda y} d y-D+c \lambda\right] \\
& +\gamma\left(k_{2}+\varepsilon_{3} e^{-\lambda t}\right)-(d+\delta)\left(k_{3}+\varepsilon_{5} e^{-\lambda t}\right) \\
:= & I_{5}(\lambda) .
\end{aligned}
$$

For $\lambda$ sufficiently small, by (29), we see that $\gamma\left(k_{2}+\varepsilon_{3}\right)-(d+$ $\delta)\left(k_{3}+\varepsilon_{5}\right)<0$ implies that $I_{5}(0)<0$ and there exists $\lambda_{6}^{*}>0$ such that $P_{3}(t) \leq I_{5}(\lambda)<0$ for all $\lambda \in\left(0, \lambda_{6}^{*}\right)$.

Clearly, for all $\lambda \in\left(0, \min \left\{\lambda_{i}^{*}, i=1, \ldots, 6\right\}\right), P_{i}(t) \leq 0(i=$ $1,2,3)$. This completes the proof.

Lemma 8. $\underline{\Phi}(t)=(\phi(t), \varphi(t), \psi(t))$ is a lower solution of system (10).

Proof. Denote

$$
\begin{aligned}
Q_{1}(t)= & D \int_{\mathbb{R}} J(t-y) \underline{\phi}(y) d y-D \underline{\phi}(t)-c \underline{\phi}^{\prime}(t)-d \underline{\phi}(t) \\
& +a \underline{\varphi}(t), \\
Q_{2}(t)= & D \int_{\mathbb{R}} J(t-y) \underline{\varphi}(y) d y-D \underline{\varphi}(t)-c \underline{\varphi}^{\prime}(t)
\end{aligned}
$$

$$
\begin{aligned}
&+\frac{\beta[A / d-\bar{\phi}(t)-\underline{\varphi}(t)-\bar{\psi}(t)] \underline{\varphi}(t-c \tau)}{1+\alpha \underline{\varphi}(t-c \tau)} \\
&-(d+\gamma+a) \underline{\varphi}(t), \\
& Q_{3}(t)=D \int_{\mathbb{R}} J(t-y) \underline{\psi}(y) d y-D \underline{\psi}(t)-c \underline{\psi}^{\prime}(t) \\
&+\gamma \underline{\varphi}(t)-(d+\delta) \underline{\psi}(t) .
\end{aligned}
$$

If $t \leq t_{2}, \phi(t)=0$. It is easy to see that $Q_{1}(t)=a \varphi(t) \geq 0$.

If $t>t_{2}, \bar{\phi}(t)=k_{1}-\varepsilon_{2} e^{-\lambda t}$ and $\varphi(t)=k_{2}-\varepsilon_{4} e^{-\bar{\lambda} t}$. Then, we have

$$
\begin{aligned}
Q_{1}(t)= & \varepsilon_{2} e^{-\lambda t}\left[-D \int_{\mathbb{R}} J(y) e^{\lambda y} d y+D-c \lambda\right] \\
& -d\left(k_{1}-\varepsilon_{2} e^{-\lambda t}\right)+a\left(k_{2}-\varepsilon_{4} e^{-\lambda t}\right) .
\end{aligned}
$$

For $\lambda$ sufficiently small, $-d\left(k_{1}-\varepsilon_{2}\right)+a\left(k_{2}-\varepsilon_{4}\right)>0$ implies that $Q_{1}(0)>0$ and there exists $\lambda_{7}^{*}>0$ such that $Q_{1}(t)>0$ for all $\lambda \in\left(0, \lambda_{7}^{*}\right)$.

If $t \leq t_{4}, \varphi(t)=0$ and $\varphi(t-c \tau)=0$. Hence, $Q_{2}(t)=0$.

If $t>t_{4}, \varphi(t)=k_{2}-\varepsilon_{4} e^{-\lambda \tau}$. Noting that $\varphi(t-c \tau) \geq$ $k_{2}-\varepsilon_{4} e^{-\lambda t}, \bar{\phi}(t) \leq k_{1}+\varepsilon_{1} e^{-\lambda \tau}$ and $\bar{\psi}(t) \leq k_{3}+\varepsilon_{5} e^{-\lambda t}$. We obtain that

$$
Q_{2}(t) \geq \varepsilon_{4} e^{-\lambda t}\left[-D \int_{\mathbb{R}} J(y) e^{\lambda y} d y+D-c \lambda\right]+I_{6}(\lambda),
$$

where

$$
\begin{aligned}
I_{6}(\lambda)= & \left(\beta \left[\frac{A}{d}-k_{1}-\varepsilon_{1} e^{-\lambda t}-k_{2}+\varepsilon_{4} e^{-\lambda t}-k_{3}\right.\right. \\
& \left.\left.\quad-\varepsilon_{5} e^{-\lambda t}\right]\left(k_{2}-\varepsilon_{4} e^{-\lambda t}\right)\right) \\
& \times\left(1+\alpha\left(k_{2}-\varepsilon_{4} e^{-\lambda t}\right)\right)^{-1} \\
& -(d+\gamma+a)\left(k_{2}-\varepsilon_{4} e^{-\lambda t}\right) .
\end{aligned}
$$

By (29), we have $I_{6}(0)>0$. Accordingly, for $\lambda$ sufficiently small, there exists $\lambda_{8}^{*}>0$ such that $Q_{2}(t) \geq I_{6}(\lambda)>0$ for all $\lambda \in\left(0, \lambda_{8}^{*}\right)$.

If $t \leq t_{6}, \psi(t)=0$. Then, we have $Q_{3}(t)=\gamma \varphi(t) \geq 0$.

If $t>t_{6}, \psi(t)=k_{3}-\varepsilon_{6} e^{-\lambda t}$ and $\varphi(t)=k_{2}-\varepsilon_{4} e^{-\lambda t}$. We obtain that

$$
\begin{aligned}
Q_{3}(t)= & \varepsilon_{6} e^{-\lambda t}\left[-D \int_{\mathbb{R}} J(y) e^{\lambda y} d y+D-c \lambda\right] \\
& +\gamma\left(k_{2}-\varepsilon_{4} e^{-\lambda t}\right)-(d+\delta)\left(k_{3}-\varepsilon e^{-\lambda t}\right) .
\end{aligned}
$$

For $\lambda$ sufficiently small, then, by (29), it is readily seen that $Q_{3}(0)>0$ and there exists $\lambda_{9}^{*}>0$ such that $Q_{3}(t)>0$ for all $\lambda \in\left(0, \lambda_{9}^{*}\right)$.

Obviously, for all $\lambda \in\left(0, \min \left\{\lambda_{7}^{*}, \lambda_{8}^{*}, \lambda_{9}^{*}\right\}\right), Q_{i}(t) \geq 0(i=$ $1,2,3)$. This completes the proof. 
Applying Lemmas 2-8 and Schauder's fixed point theorem, we know that if $\mathscr{R}_{0}>1$ and $a / d+\gamma /(d+\delta)<1$, system (9) has a traveling wave solution with speed $c>c^{*}$ connecting the steady states $(0,0,0)$ and $\left(k_{1}, k_{2}, k_{3}\right)$. Accordingly, we have the following conclusion.

Theorem 9. Let $\mathscr{R}_{0}>1$. Assume that (A1) and $a / d+\gamma /(d+$ $\delta)<1$ hold. For every $c>c^{*}$, system (3) always has a traveling wave solution with speed $c$ connecting the disease-free steady state $E^{0}(A / d, 0,0)$ and the endemic steady state $E^{*}\left(S^{*}, I^{*}, R^{*}\right)$.

\section{Concluding Remark}

In this paper, we have discussed a delayed SIRS infectious disease model with nonlocal diffusion and nonlinear incidence. By constructing a pair of upper-lower solutions and using Schauder's fixed point theorem, we investigated the existence of a traveling wave solution connecting the diseasefree steady state $E^{0}$ and the endemic steady state $E^{*}$. We now study the influence of the nonlocal diffusion terms and time delay describing the incubation period on the spreading speed $c^{*}$. From the second equation of system (3), we have a linearized equation at $E^{0}$ that takes the form

$$
\begin{aligned}
c I^{\prime}(\xi)= & D[J * I(\xi)-I(\xi)]+\frac{A \beta}{d} I(\xi-c \tau) \\
& -(d+\gamma+a) I(\xi) .
\end{aligned}
$$

Letting $I(\xi)=e^{\lambda \xi}$ yields the following characteristic equation:

$$
\begin{aligned}
\Delta(\lambda, c)= & D \int_{\mathbb{R}} J(y)\left[e^{-\lambda y}-1\right] d y-c \lambda+\frac{A \beta}{d} e^{-\lambda c \tau} \\
& -(d+\gamma+a) .
\end{aligned}
$$

By direct calculations we have

$$
\begin{aligned}
\frac{\mathrm{d} c^{*}}{\mathrm{~d} D} & =\frac{\int_{\mathbb{R}} J(y)\left[e^{-\lambda_{*} y}-1\right] d y}{\lambda_{*}\left(1+(A \beta \tau / d) e^{-\lambda_{*} c^{*} \tau}\right)}>0, \\
\frac{\mathrm{d} c^{*}}{\mathrm{~d} \tau} & =-\frac{A \beta c^{*}}{d e^{-c^{*} \lambda_{*} \tau}+A \beta \tau}<0 .
\end{aligned}
$$

It is easy to show that the spreading speed $c^{*}$ is monotonically increasing for the nonlocal diffusion rate $D$ and is monotonically decreasing for the time delay $\tau$.

\section{Conflict of Interests}

The authors declare that there is no conflict of interests regarding the publication of this paper.

\section{Acknowledgments}

This work was supported by the National Natural Science Foundation of China (nos. 11371368, 11071254), the Natural Science Foundation of Hebei Province of China under Grant (no. A2014506015), the Natural Science Foundation for
Young Scientists of Hebei Province (no. A2013506012), and the Science Research Foundation of Mechanical Engineering College (nos. YJJXM12010, YJJXM13008).

\section{References}

[1] Q. Gan, R. Xu, and P. Yang, "Travelling waves of a delayed SIRS epidemic model with spatial diffusion," Nonlinear Analysis, vol. 12, no. 1, pp. 52-68, 2011.

[2] K. B. Blyuss, "On a model of spatial spread of epidemics with long-distance travel," Physics Letters A: General, Atomic and Solid State Physics, vol. 345, no. 1-3, pp. 129-136, 2005.

[3] M. Cui, T. Ma, and X. Li, "Spatial behavior of an epidemic model with migration," Nonlinear Dynamics, vol. 64, no. 4, pp. 331-338, 2011.

[4] Y. Lou and X. Zhao, "A reaction-diffusion malaria model with incubation period in the vector population," Journal of Mathematical Biology, vol. 62, no. 4, pp. 543-568, 2011.

[5] Z. Wang, W. Li, and S. Ruan, "Travelling wave fronts in reactiondiffusion systems with spatio-temporal delays," Journal of Differential Equations, vol. 222, no. 1, pp. 185-232, 2006.

[6] P. Weng and X. Zhao, "Spreading speed and traveling waves for a multi-type SIS epidemic model," Journal of Differential Equations, vol. 229, no. 1, pp. 270-296, 2006.

[7] X. Chen, "Existence, uniqueness, and asymptotic stability of traveling waves in nonlocal evolution equations," Advances in Differential Equations, vol. 2, no. 1, pp. 125-160, 1997.

[8] J. Coville, J. Dávila, and S. Martínez, "Nonlocal anisotropic dispersal with monostable nonlinearity," Journal of Differential Equations, vol. 244, no. 12, pp. 3080-3118, 2008.

[9] P. Fife, "Some nonclassical trends in parabolic and paraboliclike evolutions," in Trends in Nonlinear Analysis, pp. 153-191, Springer, Berlin, Germany, 2003.

[10] Y. Sun, W. Li, and Z. Wang, "Entire solutions in nonlocal dispersal equations with bistable nonlinearity," Journal of Differential Equations, vol. 251, no. 3, pp. 551-581, 2011.

[11] Y. Sun, W. Li, and Z. Wang, "Traveling waves for a nonlocal anisotropic dispersal equation with monostable nonlinearity," Nonlinear Analysis A: Theory and Methods, vol. 74, no. 3, pp. 814-826, 2011.

[12] G. Zhang and Y. Wang, "Critical exponent for nonlocal diffusion equations with Dirichlet boundary condition," Mathematical and Computer Modelling, vol. 54, no. 1-2, pp. 203-209, 2011.

[13] W. T. Li, Y. J. Sun, and Z. C. Wang, "Entire solutions in the Fisher-KPP equation with nonlocal dispersal," Nonlinear Analysis, vol. 11, no. 4, pp. 2302-2313, 2010.

[14] V. Capasso and G. Serio, "A generalization of the KermackMcKendrick deterministic epidemic model," Mathematical Biosciences, vol. 42, no. 1-2, pp. 43-61, 1978.

[15] R. Xu and Z. Ma, "Stability of a delayed SIRS epidemic model with a nonlinear incidence rate," Chaos, Solitons \& Fractals, vol. 41, no. 5, pp. 2319-2325, 2009.

[16] X. Yu, C. Wu, and P. Weng, “Traveling waves for a SIRS model with nonlocal diffusion," International Journal of Biomathematics, vol. 5, no. 5, 26 pages, 2012. 


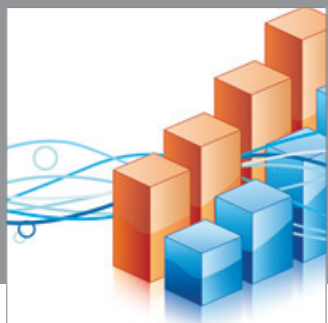

Advances in

Operations Research

mansans

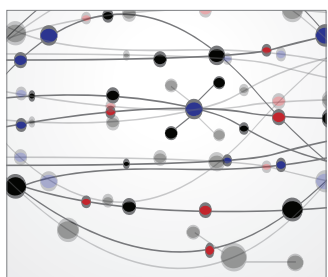

The Scientific World Journal
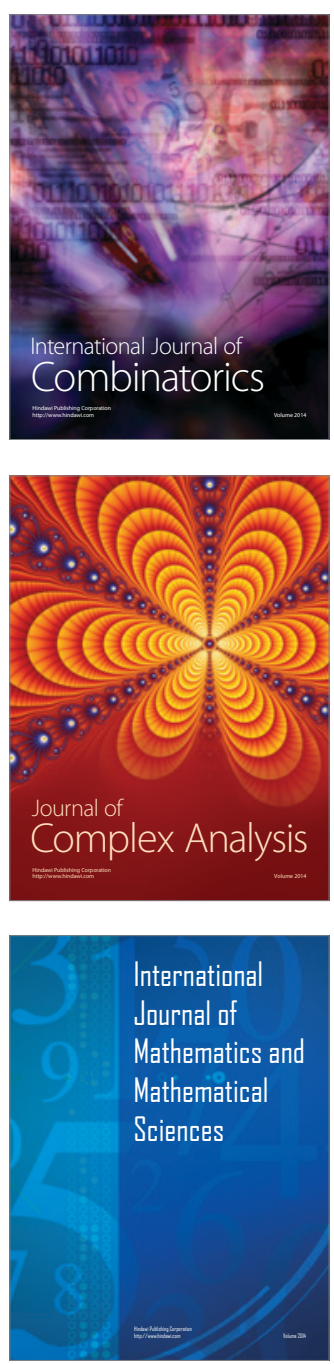
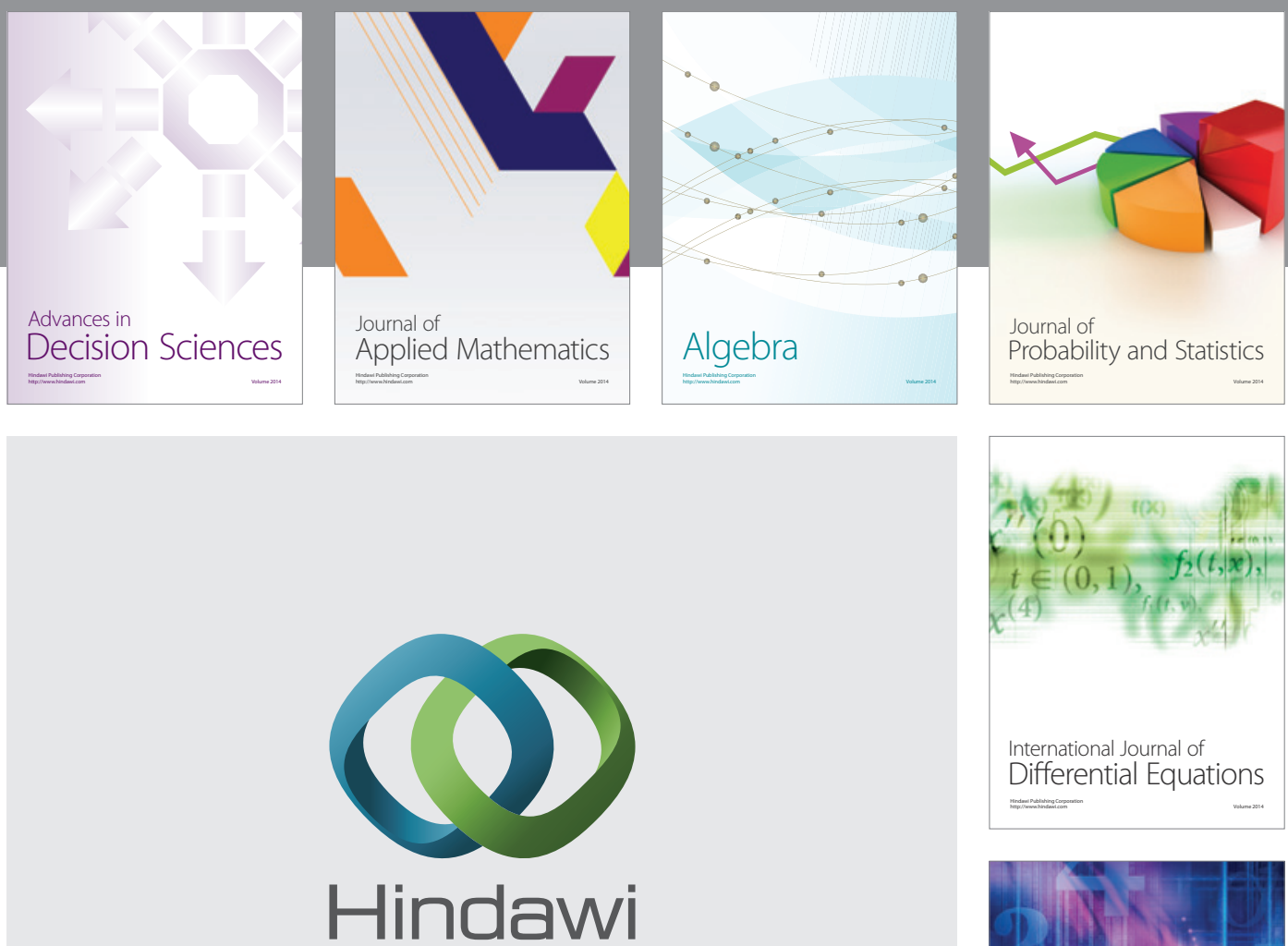

Submit your manuscripts at http://www.hindawi.com
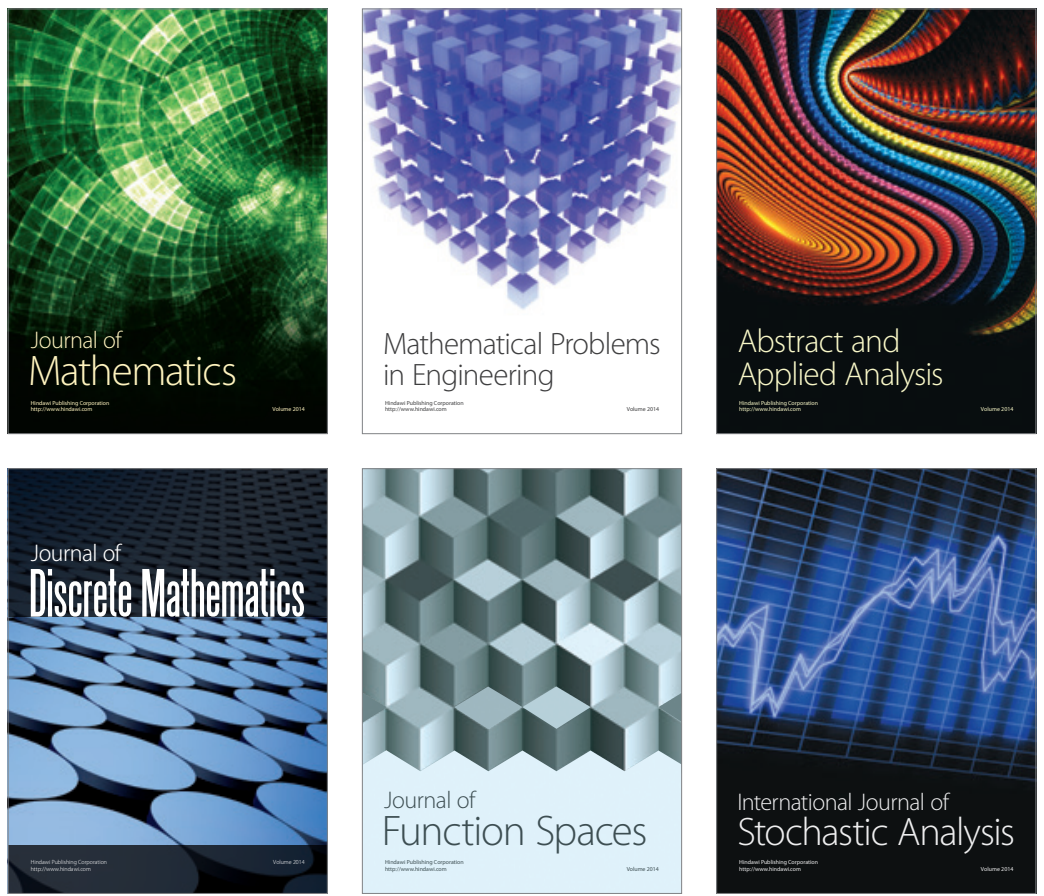

Journal of

Function Spaces

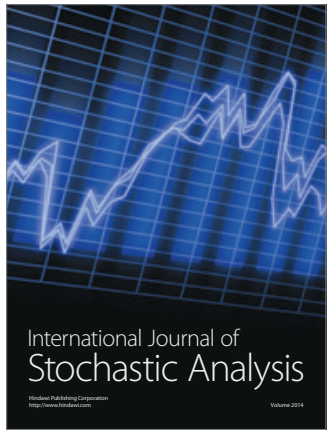

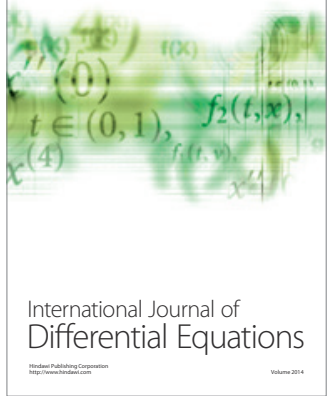
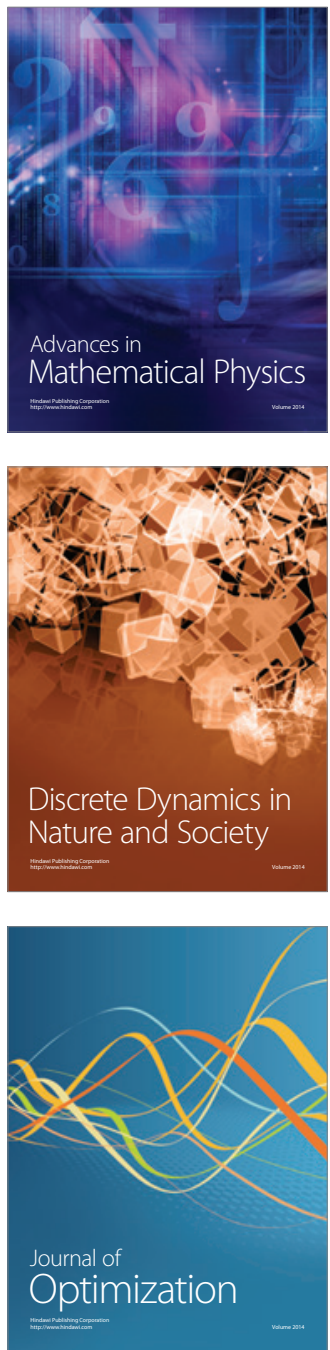